Original Research Article

\title{
The impact of therapy on the quality of life in asymptomatic patients with freshly detected hypertension
}

\author{
Satish Chandra Mishra ${ }^{1}$, Vishal Singh ${ }^{1}$, Sudeep Prakash ${ }^{2}$, Pushkar Pandey ${ }^{1}$
}

\begin{abstract}
${ }^{1}$ Department of Medicine, 7AF Hospital, Kanpur Cantt Uttar Pradesh, India

${ }^{2}$ Department of Nephrology, Army Hospital, New Delhi, India
\end{abstract}

Received: 16 July 2019

Accepted: 30 July 2019

*Correspondence to:

Dr. Vishal Singh,

Email: vishal23415@gmail.com

Copyright: (C) the author(s), publisher and licensee Medip Academy. This is an openaccess article distributed under the terms of the Creative Commons Attribution NonCommercial License, which permits unrestricted noncommercial use, distribution, and reproduction in any medium, provided the original work is properly cited.

\begin{abstract}
Background: Hypertension is an commonly encountered disease which adversely affect all aspects of quality of life (QoL). The existing studies are confounded by the presence of multiple comorbidities and inclusion of elderly, which by themselves impairs the QoL. There is thus a need to study the impact of hypertension on QoL, in isolation.

Method: This is a single center, prospective, intention to treat, observation study. The aim of the study is to evaluate the change in the QoL over six months, in newly diagnosed asymptomatic patients of hypertension. The tools used to assess the QoL included World Health Organisation's Quality of Life Questionnaire (WHOQOL- BREF) and Short Form-36 (SF-36).

Result: A total of 232 patients (172 males and 60 females) were enrolled in the study. The mean age was 44.66 years. A total of 102 patients $(43.97 \%)$ had stage- 1 and 130 patients $(56.03 \%)$ had stage- 2 hypertension. The female gender is associated with a higher likelihood of presentation with stage- 2 hypertension. The male cohort had a better baseline QoL. The desired blood pressures was achieved in $40.52 \%$. With therapy, the QoL improved significantly; sub-hoc analysis showed, the improvement was higher in males and those with stage-1 hypertension. There is an inverse relationship between the QoL and requirement for higher number of antihypertensive mediations.

Conclusions: In patients with asymptomatic primary hypertension, treatment improves all aspects of QoL. The factors adversely affecting the QoL include female gender, higher stage of hypertension, poor blood pressure control and requirement of higher numbers of antihypertensive medicine.
\end{abstract}

Keywords: Hypertension, Quality of life, Short form 36, WHOQOL

\section{INTRODUCTION}

Hypertension is a commonly encountered disease. It is a modern day epidemic and is estimated to affect in excess of one billion people worldwide. ${ }^{1}$ In the years to come, the burden of the disease is anticipated to rise. The World Health Organization (WHO) rates, hypertension as one of the most important causes of premature death worldwide. It exerts a substantial public health burden and is directly responsible for $57 \%$ of all stroke deaths and $24 \%$ of all coronary heart disease deaths in India. ${ }^{2,3}$ The QoL is a broad concept encompassing global life satisfaction including good health, adequate housing, employment, personal and family safety, education, and leisure pursuits. For matters related to health care, QoL has been applied specifically to those life concerns that are most affected by health or illness. WHO defines QoL as an individual's perception of their position in life in the context of the culture and value systems in which they live, and in relation to their goals, expectations, standards and concerns. ${ }^{4}$ Several validated and reliable questionnaires are available for assessment of QoL. These include generic health status instruments, generic illness instruments, and disease-specific instruments. These standardized indices are increasingly being used to guide individual clinical care. The SF-36 is a multipurpose, validated, short-form health survey with 36 
questions. ${ }^{5}$ It yields an 8-scale profile of functional health and well-being scores as well as psychometrically-based physical and mental health summary measures. It is a generic measure, as opposed to one that targets a specific age, disease, or treatment group. It has proven useful in surveys of general and specific populations. The WHOQOL-BREF, was developed as a short version of the WHOQOL-100 for use in situations where time is restricted. It is a person-centred, multilingual, generic instrument aimed at subjective assessment of four domains of QoL i.e. physical, psychological, social and environment.

The aim of treatment is to normalize the blood pressures and reduce the disease related complications. To achieve this many classes of antihypertensive medications are available. A vast majority of patients require two or more classes for effective blood pressure control. Though hypertension is described as a silent killer, various studies have shown that it adversely affect the QoL. ${ }^{6-8}$ Whether this impairment in the QoL is a consequence of hypertension itself, adverse effect of drugs, or presence of confounders like age is unclear. Further, a significant proportion of patients with hypertension have comorbidities like diabetes mellitus, coronary artery disease etc, which by themselves may affect the QoL. ${ }^{9}$

The relationship between the class of antihypertensive and the QoL is complex and poorly understood; the available data is contradictory. ${ }^{10,11}$ There is thus a need to study the relationship between primary hypertension and QoL in isolation, after excluding the known confounders.

\section{METHODS}

The study design is a single center, prospective, intention to treat, observation study. The aim of the study is to evaluate the changes in QoL following treatment, in newly diagnosed asymptomatic patients with primary hypertension.

Study protocol is all patients with freshly detected hypertension, reporting to the outpatient department of a tertiary care teaching hospital and fulfilling the study criteria were enrolled in the study. The patient's enrolment started in Jun 2011 and continued for the next 2 years. They were followed up for six months. The blood pressure was measured using a well standardized aneroid type sphygmomanometer with a cuff size of $12 \mathrm{x}$ $30 \mathrm{cms}$. Standard protocol was followed for measurement of the blood pressure. The first and the fifth phase of Korotkoff sound was taken as the systolic and the diastolic blood pressure. The average of two readings, taken five minutes apart was noted as the final blood pressure. Hypertension was defined as a blood pressure more than $140 / 90 \mathrm{~mm}$ of $\mathrm{Hg}$, on two or more separate occasions. These patients with hypertension were further subdivided into stage 1 and stage 2, as per Seventh Report of Joint National Committee on Prevention, Detection, Evaluation and Treatment of High Blood
Pressure (JNC 7). A detailed history, examination and investigations were carried out. The investigations included complete blood count, urine analysis, blood urea nitrogen, serum creatinine, serum sodium, serum potassium, fasting blood sugar, lipid profile, 12 lead electrocardiography and ultrasound examination of the kidneys. If secondary hypertension was suspected, appropriate evaluation was carried out; on confirmation of secondary hypertension, these patients were excluded from the study. All the patients were given detailed instructions regarding lifestyle modification. The initiation and modification of the therapy was guided by the recommendations of JNC 7. An informed written consent was obtained, and the patients were followed up on monthly interval for a period of six months. The QoL was assessed at baseline and at six months of therapy, using WHOQOL-BREF and SF-36.

\section{Inclusion criteria}

- Asymptomatic newly diagnosed patients with primary hypertension

- No pre-existing comorbidities

- Age group at the time of enrolment between 30 to 60 years.

\section{Exclusion criteria}

- Age less than 30 years or more than 60 years, at the time of enrolment

- Presence of comorbidities

- Blood pressure in Prehypertensive range

- Secondary hypertension

- Unwillingness to participate in the study.

\section{Statistical analysis}

The variables analysed included, the QoL by WHOQOLBREF and SF-36. These variables were analysed at the onset and at six months of therapy. The effects of variables like influence of gender, stage of hypertension, control of blood pressure, and number of antihypertensives required on QoL were also analysed. Qualitative variables are expressed as mean and standard deviation and discrete variables as percentage.. Paired ttest was used for within group comparison. Statistical Package for Social Sciences (SPSS) version 22 was used for statistical analysis (Machines IB. IBM SPSS Statistics for Windows, Version 22.0. IBM Corp Armonk, NY; 2013). A p-value <0.05 was considered statistically significant.

\section{RESULTS}

A total of 242 patients were enrolled in the study. Of these, 10 were excluded; 6 were lost to follow-up and 4 developed type 2 diabetes mellitus during the follow-up period. 232 patients including, 172 males $(74.14 \%)$ and 60 females $(25.86 \%)$ completed the study. The mean age of the study population is $44.66 \pm 7.45$ years. 102 patients 
$(43.97 \%)$, presented with stage I and 130 patients $(56.03 \%)$ presented with stage II hypertension. The female cohort has a significantly higher likelihood of presenting with stage-2 hypertension. The mean systolic and diastolic blood pressure for the male cohort is $159.5 \pm 13.6$ and $98.26 \pm 9.12 \mathrm{~mm} \mathrm{Hg}$; the corresponding values for the female cohort is $162 \pm 12.43$ and $99.83 \pm 9.44$ $\mathrm{mm} \mathrm{Hg}$.

At the end of six months, $40.52 \%$ patients could achieve the optimal blood pressures. It included $70(68.63 \%)$ and $24(18.46 \%)$ patients with stage-1 and stage-2 hypertension respectively (p-value: 0.001 ).

The baseline QoL indices were compared to the QoL at six months. There was a significant improvement in the mean score of all the domains in WHOQOL-BREF i.e. Domain 1 (D1- physical health), Domain 2 (D2psychological health), Domain 3(D3- social relationships), Domain 4 (D4- environment). Similarly, both the physical and mental component of SF 36 showed significant improvement at six months following the initiation of antihypertensive medications (Table 1). The improvement in QoL was significantly better in those who achieved adequate blood pressure control (Table 2).

A gender difference was noted in the QoL. The baseline QoL indices were significantly better in males for WHOQOL-BREF Domain 1, Domain 2 and mental component score of SF-36. There was no difference between the two genders, in WHOQOL-BREF Domain 3, Domain 4 and the physical component of SF-36 (Table 3). Following the institution of therapy, the QoL indices improved for both; the improvement was however significantly higher in male cohort for the physical and mental component of SF-36 (Table 4).

Amongst the patients, who presented with stage-1 and stage-2 hypertension, those who had stage-1 hypertension, had a significantly higher improvement in all QoL indices, except WHOQOL-BREF domain 3 (Table 5).

Table 1: Comparison of QoL scoring at the baseline and at 6 months of therapy.

\begin{tabular}{|c|c|c|c|c|c|c|}
\hline & \multirow{2}{*}{ Domain } & \multirow{2}{*}{ Number of patients } & \multicolumn{2}{|c|}{ Score $($ Mean \pm SD $)$ at } & \multirow{2}{*}{ P-value } & \multirow{2}{*}{ Confidence interval } \\
\hline & & & Baseline & 6th month & & \\
\hline \multirow{4}{*}{ WHO } & D1 & 232 & $59.18 \pm 17.64$ & $60.02 \pm 18.77$ & $<0.001$ & -7.81 to -5.86 \\
\hline & D2 & 232 & $59.22 \pm 17.95$ & $60.02 \pm 19.27$ & $<0.001$ & -7.90 to -5.70 \\
\hline & D3 & 232 & $62.80 \pm 21.10$ & $65.15 \pm 21.47$ & $<0.001$ & -3.03 to -1.67 \\
\hline & D4 & 232 & $60.98 \pm 19.22$ & $64.09 \pm 18.98$ & $<0.001$ & -3.80 to -2.43 \\
\hline \multirow{2}{*}{ SF-36 } & PCS & 232 & $40.25 \pm 11.12$ & $4278 \pm 11.7$ & $<0.001$ & -2.98 to -2.06 \\
\hline & MCS & 232 & $43.22 \pm 8.85$ & $46.10 \pm 9.30$ & $<0.001$ & -3.46 to -2.28 \\
\hline
\end{tabular}

Table 2: Analysis of relation between the QoL and the blood pressure control.

\begin{tabular}{|c|c|c|c|c|c|}
\hline & & Mean change scor & nonth (Mean \pm SD) & & \\
\hline & Domain & $\begin{array}{l}\text { Adequate control } \\
(\mathrm{n}=94)\end{array}$ & $\begin{array}{l}\text { Inadequate control } \\
(n=138)\end{array}$ & P-value & Confidence interval \\
\hline & D1 & $12.72 \pm 7.46$ & $2.83 \pm 4.17$ & $<0.001$ & -11.56 to -8.21 \\
\hline WHO & D2 & $12.51 \pm 8.05$ & $2.91 \pm 6.33$ & $<0.001$ & -11.56 to -7.64 \\
\hline WHO & D3 & $4.97 \pm 6.80$ & $0.55 \pm 2.66$ & $<0.001$ & -5.88 to -2.96 \\
\hline & D4 & $5.80 \pm 6.57$ & $1.28 \pm 3.12$ & $<0.001$ & -5.96 to -3.08 \\
\hline SF-36 & PCS & $5.12 \pm 3.82$ & $0.83 \pm 1.69$ & $<0.001$ & -5.11 to -3.45 \\
\hline DF-JO & MCS & $5.50 \pm 4.50$ & $0.62 \pm 1.54$ & $<0.001$ & -5.84 to -3.92 \\
\hline
\end{tabular}

Table 3: Baseline QoL index of the male and female cohorts.

\begin{tabular}{|llllll|}
\hline \multirow{2}{*}{} & Domain & \multicolumn{2}{l|}{ Mean score at baseline $($ Mean \pm SD $)$} & P-value & Confidence interval \\
\cline { 2 - 6 } & Male $(\mathbf{n}=\mathbf{1 7 2})$ & Female $(\mathbf{n = 6 0})$ & 0.005 & 2.24 to 12.35 \\
\hline \multirow{3}{*}{ WHO } & D1 & $61.07 \pm 17.58$ & $53.77 \pm 16.79$ & 0.012 & -1.10 to 9.42 \\
& D2 & $60.30 \pm 18.00$ & $56.13 \pm 17.60$ & 0.12 & -4.84 to 7.37 \\
\cline { 2 - 6 } & D3 & $63.13 \pm 21.44$ & $61.87 \pm 20.23$ & 0.683 & -2.23 to 8.96 \\
\hline \multirow{2}{*}{ SF-36 } & D4 & $61.85 \pm 19.41$ & $58.48 \pm 18.62$ & 0.069 & -.2287 to 6.30 \\
\hline & PCS & $41.04 \pm 11.12$ & $38.00 \pm 10.93$ & 0.012 & 0.83 to 6.46 \\
\hline
\end{tabular}


Table 4: The mean QoL score at the baseline and at 6 months of therapy in the male and female cohort.

\begin{tabular}{|c|c|c|c|c|c|}
\hline & \multirow[t]{2}{*}{ Domain } & \multicolumn{2}{|c|}{$\begin{array}{l}\text { Mean change score at } 6^{\text {th }} \text { month } \\
(\text { Mean } \pm \text { SD) }\end{array}$} & \multirow[t]{2}{*}{ P-value } & \multirow[t]{2}{*}{ Confidence interval } \\
\hline & & Male $(n=172)$ & Female $(n=60)$ & & \\
\hline \multirow{4}{*}{ WHO } & D1 & $7.30 \pm 7.76$ & $6.28 \pm 6.77$ & 0.478 & -1.33 to 2.84 \\
\hline & D2 & $7.34 \pm 8.52$ & $5.23 \pm 8.30$ & 0.095 & -.37 to 4.60 \\
\hline & D3 & $2.55 \pm 5.37$ & $1.76 \pm 4.87$ & 0.298 & -.70 to 2.27 \\
\hline & D4 & $3.35 \pm 5.64$ & $1.76 \pm 4.87$ & 0.183 & -.43 to 2.28 \\
\hline \multirow{2}{*}{ SF-36 } & PCS & $2.84 \pm 3.64$ & $1.79 \pm 2.79$ & 0.023 & .14 to 1.94 \\
\hline & MCS & $2.92 \pm 4.29$ & $1.68 \pm 2.34$ & 0.006 & .36 to 2.11 \\
\hline
\end{tabular}

Table 5: Mean change in the QoL indices from baseline between stage I and stage II hypertension.

\begin{tabular}{|c|c|c|c|c|c|}
\hline & \multirow{2}{*}{ Domain } & \multicolumn{2}{|c|}{ Mean change score at $6^{\text {th }}$ month $($ Mean \pm SD $)$} & \multirow{2}{*}{ P-value } & \multirow{2}{*}{ Confidence interval } \\
\hline & & Stage $1(n=102)$ & Stage $2(n=130)$ & & \\
\hline \multirow{4}{*}{ WHO } & D1 & $9.95 \pm 8.26$ & $4.40 \pm 5.83$ & $<0.001$ & 3.64 to 7.45 \\
\hline & D2 & $9.18 \pm 8.74$ & $4.93 \pm 7.83$ & $<0.001$ & 2.07 to 6.43 \\
\hline & D3 & $3.24 \pm 5.78$ & $1.64 \pm 4.69$ & 0.24 & 0.20 to 2.98 \\
\hline & D4 & $4.31 \pm 6.48$ & $2.17 \pm 3.93$ & 0.004 & 0.69 to 3.5 \\
\hline \multirow{2}{*}{ SF-36 } & PCS & $4.36 \pm 3.94$ & $1.16 \pm 2.18$ & $<0.001$ & 2.34 to 4.0 \\
\hline & MCS & $4.31 \pm 4.71$ & $1.26 \pm 2.44$ & $<0.001$ & 2.03 to 4.06 \\
\hline
\end{tabular}

Figure 6: Relationship between the number of classes of antihypertensive medications required and the QoL indices.

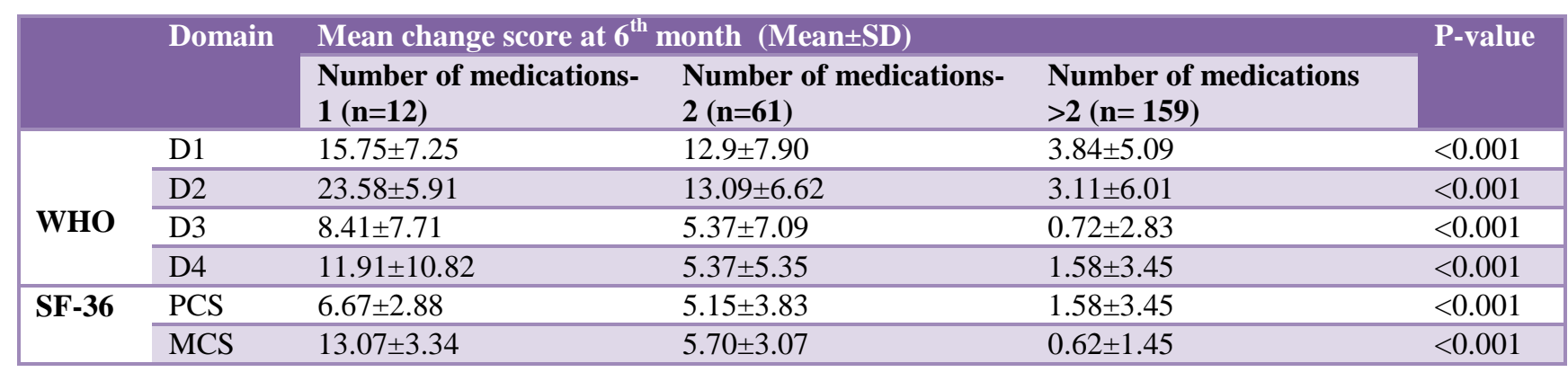

The relationship between the number of classes of antihypertensive medications required to control the blood pressures and the QoL indices was also explored. 12 patents required 1 class, 61 required 2 classes and 159 required 3 or more classes of antihypertensive medication. The patients requiring 3 or more classes of antihypertensives had significantly worse QoL indices as compared to those requiring 2 or less classes of antihypertensives (Table 6).

\section{DISCUSSION}

Hypertension is a common, potentially treatable, chronic disease affecting a large proportion of world's population. Over the years a wide range of mediations have been discovered and approved for treatment of this disease. An important, but often neglected, aspect of the disease, is the QoL. Studies in the past have shown that the QoL is worse in patients of hypertension when compared with the general population. ${ }^{8}$ The aim of the study is to assess the impact of hypertension and its treatment, on the various aspects of the QoL. The existing studies are confounded by factors like presence of comorbidities and extremes of age. In an endeavour to obviate these confounders, the study was designed to include asymptomatic patients with freshly detected primary hypertension. Patients having pre-existing comorbidities were excluded from the study. The studies in the past have shown that the minimum time for the medications to make a positive impact on the QoL is six months. ${ }^{12,13}$ Age is a major confounder affecting the QoL in patients with hypertension. ${ }^{6}$ It is not known, whether this is because of age in itself, poor social support, poor financial status, or the presence of age related comorbidities like osteoarthritis, diabetes mellitus, coronary artery disease etc.

In order to mitigate these confounders, authors excluded those above the age of 60 years from the study. Of the 232 patients, $102(43.97 \%)$ had stage I and $130(56.03 \%)$ 
had stage II hypertension. As compared to the males the females, have a higher likelihood of presentation with stage-2 hypertension. The mean baseline QoL scores in

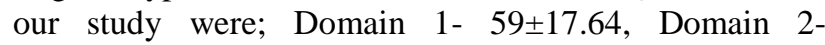

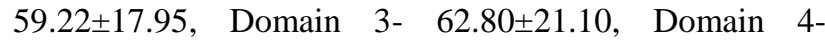
$60.98 \pm 19.22$. authors noted a higher baseline QoL as compared to earlier studies.

This was due to younger age profile, absence of comorbidities and better financial and educational background of the study population. The baseline QoL for few domains was better in males as compared to female hypertensive patients. The lower QoL in the female is likely a consequence of prevalent social prejudices. Similar findings were observed by earlier studies. ${ }^{6}$ Adequate blood pressure could be achieved in 94 (40.52\%) patients. As compared to those with stage-2 hypertension, a higher proportion of those with stage-1 hypertension, achieved adequate blood pressure control.

The baseline QoL was better in those with stage-1 hypertension, compared with those with stage-2 hypertension. This highlights the fact that the severity of hypertension has a direct negative effect on the QoL. Similarly, after the treatment, the improvement in all aspect of QoL was significantly better in those with stage1 , as compared to stage- 2 hypertensive.

This is due to higher requirement of medication and higher prevalence of suboptimal blood pressure control in patients with stage-2 hypertension. The study thus showed, not only do patients with stage-1 hypertension have a better baseline QoL, the improvement following the therapy is also significantly better. Existing studies have demonstrated, optimal blood pressure control translates in to better QoL. ${ }^{6,14,15}$ To analyse this, the study population was divided in to two cohorts; adequately controlled and inadequately controlled blood pressures. The cohort with adequate blood pressure control showed higher improvement in all domains of WHOQOL-BREF and SF-36. This suggests that the presence of hypertension in itself causes deterioration in the QoL and the institution of therapy ameliorates it. The relationship between the class of antihypertensive medication and QoL is complex and often contradictory. ${ }^{6,10,11,15}$ Further, most of the studies have shown that irrespective of the class of medication, a reduction of blood pressure improves the QoL in these patients. In light of this, authors did not analyse the relation between the class of antihypertensive medication and QoL.

The relationship between therapy, QoL and the gender was analysed. Male gender is associated with better outcome in the physical and mental component of SF-36. This is in discordance with the previous studies. ${ }^{16} \mathrm{~A}$ vast majority of patients with hypertension require multiple classes of antihypertensive to control their blood pressures. Existing studies have shown, existence of an inverse relationship between the number of class of antihypertensives required and the QoL. ${ }^{17,18}$
In our study, authors noted that the subgroup of patients requiring 3 or more classes of medication fare worse, when compared to those requiring less. Whether this is due to adverse effect of the drug, drug interaction, higher prevalence of stage- 2 hypertension, poorer blood pressure control or a combination of all these factors, is not known.

The major limitation of the study is small sample size.

\section{CONCLUSION}

In freshly detected asymptomatic patients with primary hypertension, treatment improves all aspect of QoL. The factors adversely affecting the QoL include, female gender, stage of hypertension, adequacy of blood pressure control and the number of classes of antihypertensives required. The baseline QoL for female hypertensives is lower as compared to males. Following the therapy, the QoL improved in both the genders; the improvement was however significantly more in males. There is a direct correlation between the stage of hypertension and the QoL; both the baseline QoL and the improvement following therapy was better in those with stage-1 hypertension as compared to those with stage-2 hypertension. An optimal blood pressure control translates into higher improvement in the QoL indices. There is an inverse relationship between the QoL and the number of classes of antihypertensive medications required to achieve optimal blood pressure control.

\section{ACKNOWLEDGEMENTS}

The authors acknowledge the sincere efforts of all the paramedical and support staff of the hospital without which the study would not have been possible.

Funding: No funding sources

Conflict of interest: None declared

Ethical approval: The study was approved by the Institutional Ethics Committee

\section{REFERENCES}

1. Chobanian AV, Bakris GL, Black HR, Cushman WC, Green LA, Izzo JL et al. The Seventh Report of the Joint National Committee on Prevention, Detection, Evaluation, and Treatment of High Blood Pressure: The JNC 7 Report. JAMA. 2003;289(19):2560-72.

2. Reddy KS, Shah B, Varghese C, Ramadoss A. Responding to the threat of chronic diseases in India. Lancet. 2005;366(9498):1744-9.

3. Gupta R. Trends in hypertension epidemiology in India. J Human Hypertension. 2004 Feb;18(2):73-8.

4. WHOQOL Group. Development of the WHOQOL: Rationale and current status. Int $\mathrm{J}$ Mental Health.1994;23(3):24-56.

5. Jenkinson C, Wright L, Coulter A. Criterion validity and reliability of the SF-36 in a population sample. Qual Life Res. 1994;3(1):7-12. 
6. Youssef RM, Moubarak II, Kamel MI. Factors affecting quality of life of hypertensive patients. East Mediterran Health J. 2005;11(1-2):109-18.

7. Poljičanin T, Ajduković D, Šekerija M, PibernikOkanović M, Metelko Ž, Mavrinac GV. Diabetes mellitus and hypertension have comparable adverse effects on health-related quality of life. BMC Public Health. 2010;10(1):12.

8. Stewart AL, Greenfield S, Hays RD, Wells K, Rogers WH, Berry SD, et al. Functional status and wellbeing of patients with chronic conditions: results from the Medical Outcomes Study. JAMA. 1989;262(7):907-13.

9. Aydemir O, Ozdemir C, Koroglu E. The impact of co-morbid conditions on the SF-36: a primary-carebased study among hypertensives. Arch Med Res. 2005;36(2):136-41.

10. Croog SH, Levine S, Testa MA, Brown B, Bulpitt CJ, Jenkins CD, et al. The effects of antihypertensive therapy on the quality of life. New England J Med. 1986;314(26):1657-64.

11. Wiklund I, Halling K, Ryden-Bergsten T, Fletcher A. Does lowering the blood pressure improve the mood? Quality-of-life results from the Hypertension Optimal (HOT) Study. Blood Press. 1997;6(6):357-64.

12. Fletcher AE, Bulpitt CJ, Chase DM, et al .Quality of life with three antihypertensive treatments. Cilazapril, atenolol, nifedipine. Hypertens. 1992;19(6):499-507.

13. Marcia A, Testa MA, Anderson RB. Nackley JF, Hollenberg NK. Quality of life and antihypertensive therapy in men-a comparison of captopril with enalapril. NEJM. 1993;328(13):907-13.

14. Roca-Cusachs A, Dalfo A, Badia X, Aristegui I, Roset M. Relation between clinical and therapeutic variables and quality of life in hypertension. J Hypertens. 2001;19(10):1913-9.

15. Li W, Liu L, Puente JG, Li Y, Jiang X, Jin S, et al. Hypertension and health-related quality of life: an epidemiological study in patients attending hospital clinics in China. J Hypertens. 2005;23(9):1667-76.

16. Staessen JA, Fagard R, Thijs L, Celis H, Arabidze GG, Birkenhäger $\mathrm{WH}$, et al. Randomised doubleblind comparison of placebo and active treatment or older patients with isolated systolic hypertension. Lancet. 1997;350(9080):757-64.

17. Roca-Cusachs A, Dalfó A, Badia X, Arístegui I, Roset M. Relation between clinical and therapeutic variables and quality of life in hypertension. J Hypertens. 2001;19(10):1913-9.

18. Materson BJ, Reda DJ, Cushman WC, Massie BM, Freis ED, Kochar MS, et al. Single-Drug Therapy for Hypertension in Men-A Comparison of Six Antihypertensive Agents with Placebo. New England J Med. 1993;328(13):914-21.

Cite this article as: Mishra SC, Singh V, Prakash S, Pandey P. The impact of therapy on the quality of life in asymptomatic patients with freshly detected hypertension. Int J Basic Clin Pharmacol 2019;8:1983-8. 